\title{
E-Business Logistics Based on the Storage of Robot Picking Mode Study
}

\author{
Binbin Fu, Juntao Li, Yiming Wei \\ School of Information, Beijing Wuzi University, Beijing, China
}

Email address:

binbin6807@163.com (Binbin Fu), Ljtletter@126.com (Juntao Li),879353329@qq.com (Yiming Wei)

To cite this article:

Binbin Fu, Juntao Li, Yiming Wei. E-Business Logistics Based on the Storage of Robot Picking Mode Study. Automation, Control and Intelligent Systems. Vol. 3, No. 3, 2015, pp. 39-43. doi: 10.11648/j.acis.20150303.13

\begin{abstract}
With the continuous development of e-business, e-business distribution center operating mode is gradually changing. Small batch and more frequency is the characteristics of the e-business. The new characteristics gives birth to new distribution center picking mode. This paper analyzes disadvantaged of the business mode of the current e-business distribution center, put forward a new type of e-business distribution center picking mode, which is based on storage e-business logistics mode order picking robot. Paper on the business model of the pattern has carried on the detailed analysis and logistics process design, and said that the mode is the trend of the development of e-business in the future.
\end{abstract}

Keywords: Warehouse Robot, E-Business Logistics, Picking Model

\section{E-Business Logistics and Present Situation}

E-business refers to using the Internet or other electronic tools(including telegraph, telephone, radio, television, fax, computer, computer network, mobile communication, etc.) based on computer network of a variety of business activities in the global business trade activity, including goods and services provider, advertisers, consumers, middlemen and related the sum of the conduct of parties.

Logistics as an important link in the process of e-business, for the physical distribution service between merchants and customers, e-business plays a vital role by efficient logistic system, reduce costs, enhance competitiveness in improve service. As more varieties, high frequency, small batch, the characteristics of e-business logistics gradually, it has high flexibility, high efficiency, high quality of service features ${ }^{[1]}$. This model not only led to the rapid development of modern logistics technology and equipment, but also makes the updating cycle of logistics mode more and more short. The key to improve the processing capacity of e-business logistics network nodes is to improve e-business logistics capability, warehousing, sorting, transport and distribution link to adopt intelligent information system and automation equipment, to improve the logistics speed and ability, to avoid the delivery do not in time, achieve finally "timing" "time limit for highly flexible service". And in the aggregate, e-business logistics mainly has the following characteristics: 1. information; 2. automation; 3. network; 4. intelligent; 5 . Flexibility ${ }^{[2]}$. These characteristics make the e-business logistics center operation different from other areas.

\section{E-Business Operation Status and Development Analysis}

The continuous development of e-business makes e-commerce industry new characteristics, such as order of many varieties, small batch, high frequency inferior, our country e-commerce is the main traditional manpower assignment on logistics distribution center in present, the high cost operation mode, low service efficiency, at the same time on the efficiency and service level can not meet the demand of this kind of e-business logistics in fast response, which makes the electricity distribution center are different from traditional logistics system, construction of electricity distribution center operations should adapt to the change of market construction more rapid response ability of the distribution center, the application of the automation equipment as well as the overall solution has to be adapted to the development of electricity industry characteristics. To the guideline of foreign Amazon which is using KIVA, put forwards a new mode of distribution center operation provides a guide to the e-business industry in our country. E-business operation need to be flexible and 
efficient logistics support system, in order to implement the limited resources to focus on the e-business enterprise real advantage areas, conform to the e-distribution center of the robot arises at the historic moment.

Distribution center based on warehouse robot has changed operation mode, the automatic, intelligent, integrated logistics center system can not only improve the operational efficiency and customer satisfaction of logistics, with more and more expensive human cost of the environment at the same time, it can saves logistics cost and development prospect of logistics mode in the long run. The model compared with the traditional manual operation mode are a lot of advantages, such as save labor resources, improve the space utilization, reduce the cost, etc., and have chosen as the main operation of distribution center link, its efficiency affects the overall operating efficiency directly, this article based on a new type of e-business storage robot distribution center operating mode, analyzed the picking operation mode and business process, the distribution center selection model suited to the characteristics of the electricity industry, and find out the main factors influencing the distribution center selection efficiency, improve the efficiency of distribution center selection operation.

The most typical points in storage system application is Amazon's warehouse, the application of the new logistics equipment KIVA AGV, people in the form of active bearing storage unit of the advanced warehouse machine, it can moves in the warehouse and fetching goods quickly and accurately, goods dispatch can be carried out in accordance with the orders for the first time at the same time, improves the efficiency of its goods delivery ${ }^{[3-5]}$. New warehouse operation mode has changed the traditional way of storage and pick, from passive to active, namely displacement of fixed shelves and warehouse the goods is not limited by geographical location, AGV controlled by software instruction fetching goods to the destination. This means that each staff do not need to change work position, it can be accurate to obtain the customer needs the goods in a very short time. The goods are effectively organized, greatly improve the efficiency of the goods accurately chosen, and can obtain the goods which customer needs in a short time, improves efficiency at the same time, and also improves security. Through practical application, Amazon has saved a maximum of 916 million dollars a year.

\section{Storage Characteristics and Robot Parameters}

Warehouse robot equipped with automatic guiding device carries along the predefined rules on the road, load shelves safety accurately, and handle in the warehouse without the need for manual handling automatically, and its power source can keep at any time, charging storage robot control the route through the upper machine, and behavior. Warehouse robot is key laboratory research and development of intelligent logistics system which can be used in the warehouse inventory, picking, dispatch independently controlled robots such as homework, namely the logistics handling car in this paper.

Warehouse robot working mode: warehouse robot receive picking and other scheduling task, automatic handling shelves along the shortest path to the side, if the robot need to turn to stop in case and spin to continue along a prescribed path, when the shelf arrive the target, the robot stopped running after spin to the target at the bottom of the racks, spin around to make the support shaft rising way of lifting shelf to a certain height, the racks of the path as stipulated operation work needs to be done, in case of need to turn, robot spin in place to ensure that static way to body rotation, shelf, and ensure the stability of the shelves, and to prevent the collision between the shelves ${ }^{[6]}$. After robot shipped to the destination within the given waiting area waiting for the next steps, when completion of the operation, the robot carries the shelves back to its place in the same way, arrived in destination, the robot stopped, also reduce the supporting shaft height in the form of spin gradually around to unload the carrying shelves, after completed, robot itself back to wait for next time scheduling tasks scheduling area.

Warehouse robot operation model of special, new, automated not only broke the traditional mode of the layout of the electricity distribution center, it also put forward higher requirements and challenges. First, in the limited channel width, robot should spin around the operation of the way of lifting shelf, second, robot turning action also take place at the crossroads turn out the way, at the same time, in order to avoid the collision problem between the steering rack, the robot needs to do to "turn the car body shelves still" and to maintain as far as small possible turning radius to finish turning action within the channel in addition, in order to minimize the robot working space. Finally, in order to guarantee the efficiency, the robot should not too low load rate, load bearing of the robot not too low robot weight at the same time try to stay low.

The mode shelves through the above analysis as well as the basic setup of the channel and so on, set the warehouse robot basic parameter requirements are shown now in table 1:

Table 1. Warehouse robot parameters.

\begin{tabular}{ll}
\hline $\begin{array}{l}\text { Warehousing project robot } \\
\text { parameters }\end{array}$ & Parameter requirements \\
\hline size & $750 * 600 * 425 \mathrm{~mm}$ \\
self-respect & $=200 \mathrm{~kg}$ \\
bearing & $300 \mathrm{~kg}$ \\
No-load speed & $1.0 \mathrm{~m} / \mathrm{s}$ \\
Loading speed & $0.5 \mathrm{~m} / \mathrm{s}$ \\
Lifting time & $5 \mathrm{~s}$ \\
Lifting way & Body spin around lifting \\
Turned to the way & Spin, body rotation shelves still \\
Turning radius & Robot steering shaft axis of longest \\
\hline
\end{tabular}

Parameters required by the work mode and warehouse robot, the robot structure design schematic diagram and the real figure, respectively, the following figure 1, as shown in figure 2: 


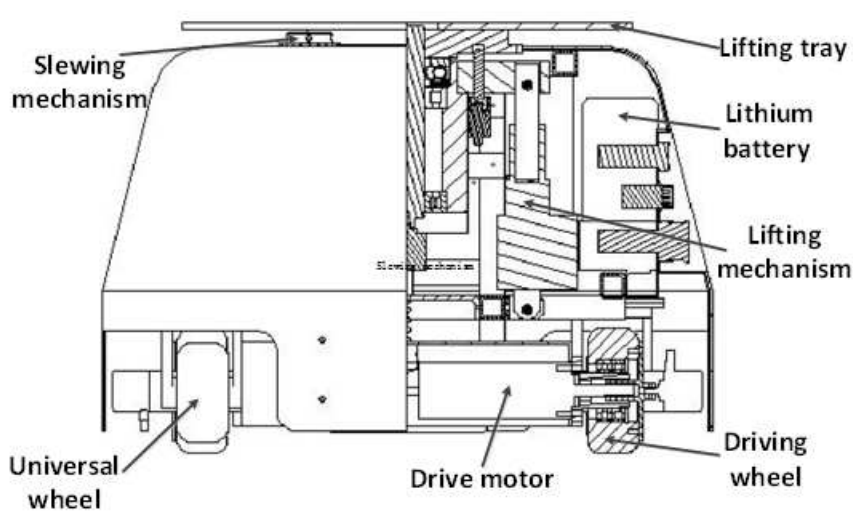

Figure 1. Warehouse robot structure design sketch.

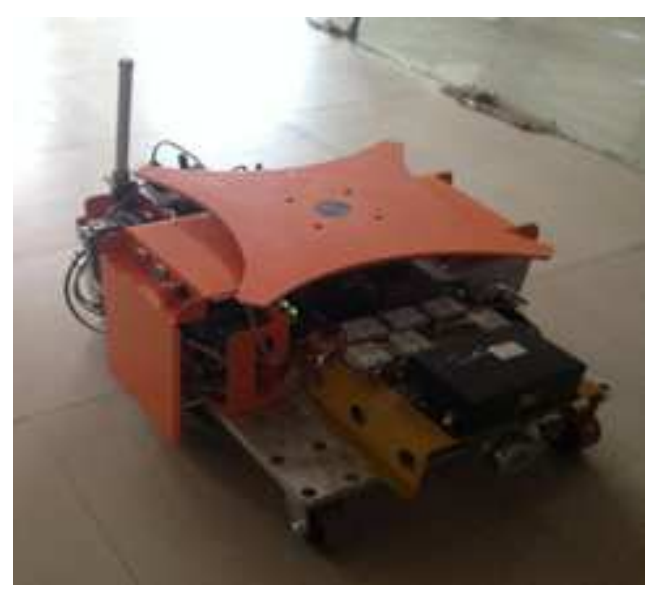

Figure 2. Warehouse robot real figure.

By the picture above, e-business logistics distribution center system based on warehouse robot operating mode requirements this kind of submersible logistics handling robot by inertial navigation and QR code for indoor positioning, implement six institutions, four-wheel for universal wheel, the middle two rounds of driving wheels in the before and after, drive to mode for the differential steering, also can realize the storage machine work together, mainly for electricity for ins and outs of logistics distribution center of the work, such as access, chosen to run in the bottom of the racks, walking, steering and in narrow space, lifting the mode to the shelves, the top has the slewing mechanism, slewing mechanism and steering mechanism of relative movement, shelves with robot collision rotation, to ensure that narrow space around the logistics handling robot with multiple laser collision sensor.

\section{E-Business Distribution Center Model Analysis}

E-business enterprise type variety, each e-business enterprise must according to their own site layout, product features, order structure choice suits own operation mode, the design of electric logistics center need to choose the suitable model according to the respective business situation, for example, the detailed scheme, process, personnel, investment estimate and so on, and adjust the corresponding plan optimization and upgrade with the development of business scale ${ }^{[7-8]}$.

E-business logistics picking based on warehouse robot is a kind of new mode. The model realized the automatic logistics imagination into reality, namely in the channel in the factory are the thousands of robot. Goods storage and distribution model for the system innovation of goods stored in removable shelves. When the goods need to be obtaining background control system automatically place, robot path to reach goods shelves, robot along the ground according to the instructions of the encoded network route to the corresponding shelf touchpad, lifting shelf again to choose the operator station ${ }^{[3]}$. Robot will be transported back to the original shelves after workers taken the goods again by storing. In this advanced warehousing logistics center stores and distribution of goods show no workers busy scene, across and carrying shelves replaced by a group of robots under computer control in an orderly way. Compared with the traditional warehousing and distribution system, the system can be one or two times higher order processing efficiency, this advantage for highly dependent on warehousing and distribution of large-scale retail and make e-business site strong attraction. The following are based on different selection of warehouse robot mode analysis and design, the main research according to the picking orders has chosen and according to the order of two kinds of operation mode, and comparing the two modes work.

\subsection{List Picking Operation Mode Analysis}

To the list picking operation mode, first is order of distribution center by the system analysis and processing, in order with the same product or similar products or order, assigned to each port with a batch of order processing to become picking list as the picking task. With picking orders generated, the system will release instruction in order shelves distribution selection box, and workers correspond to the order number and order code label. After receive the task from the cache, robot start from warehousing zone to labeling shelves completed, then carrying shelves to choose the appropriate position of the mouth, after entering the picking area by the system automatically, robot recognized chosen shelves and sending it to the specified port. Workers chosen plenty of goods according to the light and screen display specified in the corresponding box until the shelf cases chosen completely in the task ${ }^{[9]}$. Finally, goods which have chosen will be back to its place by warehousing robots, operation of storage shelves and then will complete the order which has chosen shelves to second place, the chosen orders again for packaging processing, the chosen link ends on to the next task operation.

\subsection{List Picking Operation Process Design}

Chosen according to the picking orders, first optimized according to the time window by the order processing system, namely in the specified time interval processing of a batch of orders, the number of orders for similarity comparison, classification, such as operation, aimed to improving the same 
goods in the shelf to the chosen order coverage of mouth through the handling of the orders with high similarity of goods or products in the same order to merge into the picking list, in order to reduce the extra storage robot reciprocating motion, improve the picking efficiency.

Under the picking area, operators choose picking mouth for picking orders every time, allocation chosen order based on system optimization according to each single order at the same time, and corresponding chosen box to the number of code labeling. At this point, when choosing picking box, it can order according to the characteristics of the product, such as size, volume, etc., choose the appropriate orders box to save the body material. Then, scans the shelf to system to ensure the labeling has completed, show that nearby warehouse robot which has completed labeling shelves to have taken place according to the requirement.

Put orders input system, workers will picking to optimize the personnel in the chosen terminal in the form of the bottom of the screen to start button calls the picking task, call signal nearby scheduling storage robots according to the system, robot run according to the instruction to picking preparation area in handling with shelf labeling good order to choose appropriate location, shelves, if there has robot waiting in front of the existing in the operation order area, upon the shelves, the robot to replace the original robot position, or according to the in front of the mouth will be chosen each needs first to other need this rack has chosen mouth to operation again returned to the waiting area for choose mouth scheduling to reduce the waiting time for robot.

At the same time, the system dispatch from a recent storage robots according to the chosen order information, robot recognition for shelves according to the instructions and choose the path chosen in the shelf below the lifting shelf according to certain rules, shelves according to the established rules to choose the path to picking, if already ahead in the shelves, the operation is waiting for the waiting in line to leave before a robot to the robot position. When press the start button, the screen display shows above required picking goods information, including name, quantity, location, etc., and the goods display lights up, order shelves response position indicator light is lit up at the same time, the member will be chosen to be the full amount of the goods into the corresponding order after box, press the confirm button beside the crate, the indicator light out, and at the same time the next container and the goods of the light is lit up, corresponding picking rack can be chosen to the next goods until the goods shelves, picking task has been completed, if the other chosen mouth have the shelves of goods demand, the robot run according to the given path to the next selection, waiting for the picking operation, otherwise the robot run to the store according to the established rules, robots waiting for the next scheduling tasks, such as no scheduling tasks automatically back to the empty car parking area, if robots in charging status right now, choosing paths in the charging area waiting in line to the nearest charging. Mouth shelf of all orders will be chosen when the same after completion of all tasks, robots and shelves by gravity and the number of lights on the dual induction automatic leave, and will be the order shelves to the secondary packaging area split after reduction of customer orders waiting for packaging. List will be chosen according to the operation flow chart shown in figure 3 :

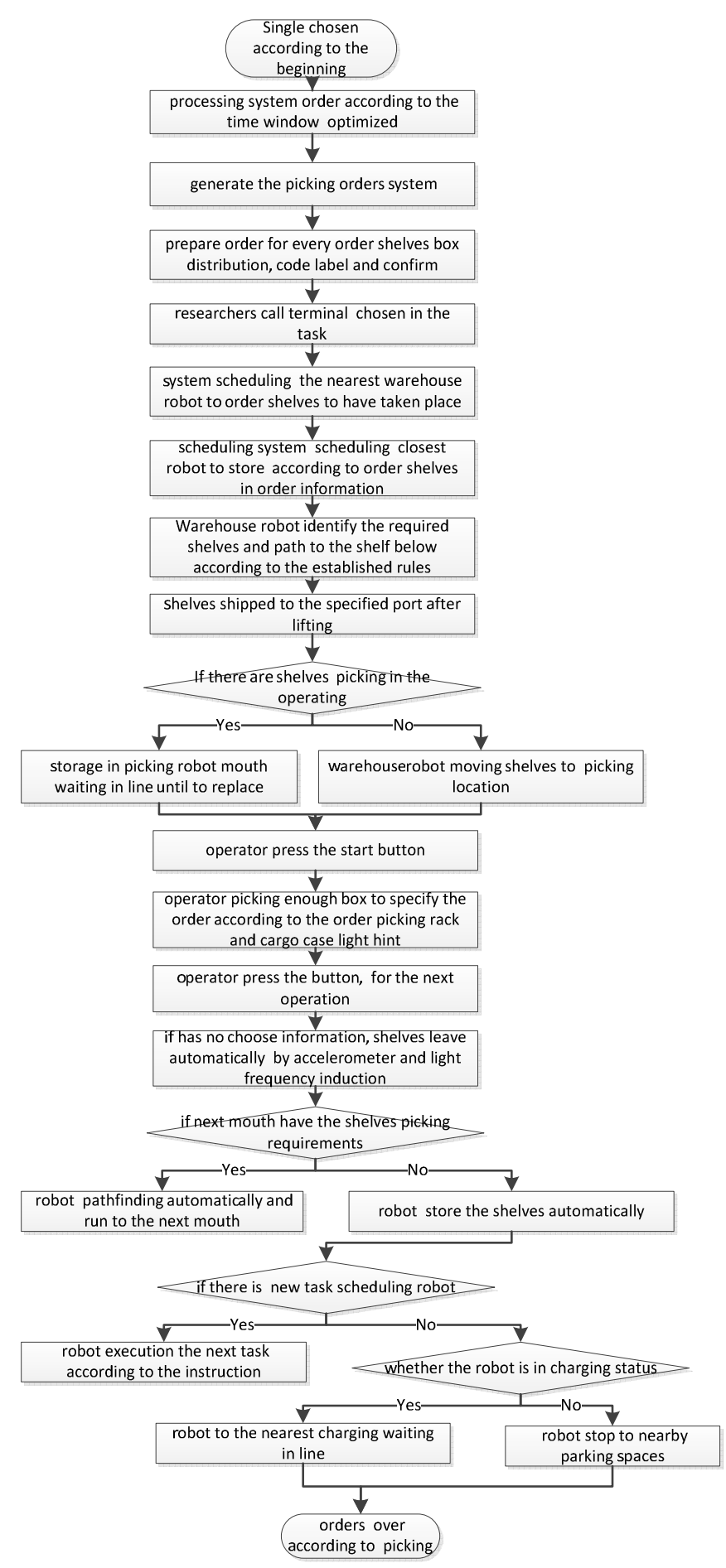

Figure 3. List will be chosen according to the operationflow chart.

\section{Conclusion}

Picking in e-business distribution center becomes an important factor in the influence of efficient in distribution center. Currently, domestic B2C e-business enterprises have 
chosen link of low degree of automation, the basic link to manual operation mode. Due to the disadvantages of artificial patterns inherent, makes the lower operational efficiency of the distribution center. The current e-business distribution center selection task for basic patterns is that chosen workers pushing a car and carry them along the storage area to walk $\mathrm{S}$ route, at the same time for dozens of order picking, choose car pushed to planting area after walking a circle, then picking orders for two points for a single order.

Picking system based on warehouse robot artificial model has more advantage. Moreover, when the shelves number and order quantity larger makes operation mode in the distribution center area reflect its advantage more, with the enlargement of the scale, the efficiency of the chosen system advantage will also expand. In nowadays e-commerce business development, customer experience becomes the important factor of e-business in an impregnable position ${ }^{[10]}$. And to improve the customer experience and the efficiency of distribution center operation, especially the efficiency of the low efficiency of the chosen segment is crucial at present.

As a new operation mode, the prophase of fixed investment becomes necessary, the investment will be relatively reduced after a long period of cost-sharing. Currently, labor cost is growing in constant, accumulated over a long period of time, the investment will very much. Traditional model needs to put in storage, storage, picking points to distinguish between equipment, while the picking system based on warehouse robot can do the same area and basic equipment. It will relatively reduce investment of equipment and site input, model for same equipment can improve the utilization rate of equipment. Automated operation pattern has always been diligent direction of the businesses and industries, it not only makes the process simplification, stylized, and the walking reduce operator's mechanized handling action mode makes the process more human. Therefore, e-business based on the storage of robot complied with the trend of the development of the distribution center operation system.

\section{Acknowledgements}

This paper is supported by the Funding Project for Technology Key Project of Municipal Education Commission of Beijing (ID:TSJHG201310037036); Funding
Project for Beijing key laboratory of intelligent logistics system ;Funding Project of Construction of Innovative Teams and Teacher Career Development for Universities and Colleges Under Beijing Municipality (ID:IDHT20130517), and Beijing Municipal Science and Technology Project (ID:Z131100005413004);Funding Project for Beijing philosophy and social science research base specially commissioned project planning (ID:13JDJGD013).

\section{References}

[1] Programme. E-commerce "twelfth five-year" development plan[EB/OL],http://www.miit.gov.cn/n11293472/n11293832/n 11293907/n11368223/14527814.html. 2012-03-27.

[2] X.N. Wang. E-commerce logistics management[M].Beijing: Cambridge university press, 2012, 20-21.

[3] ADI company. Kiva Systems will use Blackfin navigation intelligent robots are employed in the warehouse http://www.gongkong.com/webpage/solutions/200910/200910 1611212700007.Htm.2009-10-16.

[4] M.Zhang. Parts of new warehouse distribution center layout planning study[D]. Jilin university, 2014.

[5] X.T. Wang. product distribution center planning and research about Qingdao Eurasian jewelry company[D] Ocean university of China, 2013

[6] PETERSEN, C.G. An evaluation of order picking routing policies[J]. International Journal of Operations \&Production Management, 1997, 17(11):1098-1111.

[7] CHOE, K., SHARP, GP., Serfozo, R.S.. Aisle-based order pick systems with batching, zoning and sorting[C]. Progress in Materail Handing Research, 1992, 245-276.

[8] DE KOSTER R., NEUTEBOOM A.J.. The Logistics of Supermarket Chains[M]. Elsevier,2001, Doetinchem.

[9] VAN DEN BERG J.P., GADEMANN A.J.R.N.. Simulation study of an automated storage/retrieval system[J]. International Journal of Production Research, 2000, 38:1339-1356.

[10] Clark, C. 2005. Probabilistic road map sampling strategies for multi-robot motion planning. Journal of Robotics and Autonomous Systems 2005, 53(3-4): 244-264. 